\title{
Prognostic significance of Ki67 expression and the derived neutrophil-lymphocyte ratio in nasopharyngeal carcinoma
}

This article was published in the following Dove Press journal: Cancer Management and Research

\author{
Liang Zhao \\ Haojun Chen ${ }^{2}$ \\ Bin $\mathrm{Hu}^{3}$ \\ Huan Zhang' \\ Qin Lin'
}

'Department of Radiation Oncology, Xiamen Cancer Hospital, The First Affiliated Hospital of Xiamen University, Teaching Hospital of Fujian Medical University, Xiamen, China; ${ }^{2}$ Department of Nuclear Medicine \& Minnan PET Center, Xiamen Cancer Hospital, The First Affiliated Hospital of Xiamen University, Teaching Hospital of Fujian Medical University, Xiamen, China; ${ }^{3}$ Department of Laboratory Medicine, The First Affiliated Hospital of Xiamen University, Teaching Hospital of Fujian Medical University, Xiamen, China
Correspondence: Qin Lin Department of Radiation Oncology, Xiamen Cancer Hospital, The First Affiliated Hospital of Xiamen University, Teaching Hospital of Fujian Medical University, 55 Zhenhai Road, Xiamen, Fujian 361003, China

$\mathrm{Tel}+865922139152$

Fax +865922137189

Email linqin05@163.com
Purpose: To investigate the association of pretreatment Ki67 expression and the derived neutrophil-lymphocyte ratio (dNLR) with clinical outcomes in nasopharyngeal carcinoma (NPC). Patients and methods: For the study, 46 patients diagnosed with NPC at our hospital were recruited between April 2013 and December 2015. All patients were histologically confirmed to have non-keratinizing undifferentiated NPC. The expression of Ki67 proteins in NPC tissue was analyzed immunohistochemically, and the dNLR was assessed in the peripheral blood, both before treatment. Stage I and II disease was treated with radiotherapy with or without concurrent chemotherapy, and stage III and IV disease was treated with cisplatin-based radiochemotherapy and neoadjuvant chemotherapy regimens.

Results: Forty-five of the 46 patients met the criteria, and the median follow-up period was 41 months (15-56 months). The cutoff values for Ki67 and dNLR were $77.5 \%$ and $2.01 \%$, respectively. The 3-year overall survival (OS) and progression-free survival rates in the high versus low Ki67 expression groups were $62.5 \%$ vs. $93.1 \%(P=0.009)$ and $56.3 \%$ vs. $93.1 \%$ $(P=0.003)$, respectively. The 3 -year OS rate of patients with high dNLR vs. low dNLR was $64.3 \%$ vs. $90.3 \%(P=0.023)$. In the Cox risk ratio model, Ki67 expression and dNLR were independent prognostic factors for OS. Patients were then divided into three groups based on Ki67 expression and dNLR (high risk, both factors were high; intermediate risk, one factor was high; and low risk, neither factor was high). The 3 -year OS rates were $20 \%, 85 \%$, and $95 \%$ for the high, intermediate, and low risk groups, respectively $(P<0.001)$.

Conclusion: Pretreatment Ki67 and dNLR levels can be used as independent prognostic markers in NPC, and elevated values are associated with poor prognosis. Concurrently, high Ki67 expression and dNLR predict a significantly adverse outcome.

Keywords: nasopharyngeal carcinoma, Ki67, dNLR, prognosis

\section{Introduction}

Nasopharyngeal carcinoma (NPC) is a common malignant tumor of the head and neck. The undifferentiated subtype of NPC is endemic in southern China, with the peak incidence approaching 30 cases per $100,000 .{ }^{1}$ Due to developments in radiotherapy and the wide application of chemotherapy, the 5-year survival rate has improved dramatically. However, recurrence and distant metastases remain key challenges for cancer treatment failure. ${ }^{2}$ Therefore, the identification and evaluation of pretreatment biomarkers could help predict prognosis.

Ki67, also known as antigen KI-67 or MKI67, is a protein encoded by the MKI67 gene. Ki67 is required for maintaining individual mitotic chromosomes dispersed in the cytoplasm after nuclear envelope disassembly. ${ }^{3}$ The Ki67 protein is present in all 
active phases of the cell cycle but is absent in quiescent cells. ${ }^{4}$ Thus, Ki67 is an excellent marker of cell proliferation, and as such, there is abundance of research on $\mathrm{Ki67}$ in various cancers, especially lymphoma and breast, lung, and brain cancers. ${ }^{5,6}$ For instance, Ki67 levels are used to predict the efficacy of chemotherapy and hormone receptor blockade in estrogen receptor-positive breast cancer. ${ }^{7,8}$ Higher Ki67 expression is associated with poor overall survival (OS) and disease-free survival (DFS) in patients with primary breast cancer. ${ }^{9}$ However, in other cancers, such as colorectal cancer, high Ki67 expression is an independent favorable prognostic marker. ${ }^{10}$ Therefore, the role of Ki67 as a prognostic marker in different tumors is controversial, and there have been only a few studies on its potential prognostic role in NPC.

While it is known that tumor development has a genetic basis, recent studies have revealed that inflammatory response plays an indispensable role in carcinogenesis and progression. ${ }^{11,12}$ Consequently, some blood parameters have been reported to reflect the link between inflammation and cancer, such as neutrophils, lymphocytes, albumin, lactate dehydrogenase (LDH), and C-reactive protein (CRP). The neutrophil to lymphocyte ratio (NLR) has been linked to inflammatory status and clinical outcomes in several cancers, including NPC. ${ }^{13-15}$ Proctor et al evaluated the potential prognostic value of a novel biomarker, the derived NLR (dNLR), in various human cancers and showed that the prognostic value of the dNLR is similar to that of the NLR. ${ }^{16}$ The dNLR has the advantages of being inexpensive and easy to calculate, and it can be routinely measured in day-to-day clinical practice.

The aim of the present study was to investigate the prognostic value of Ki67 and dNLR on OS and progression-free survival (PFS) in patients with non-keratinizing undifferentiated NPC.

\section{Patients and methods Patients and tissue samples}

In this study, we collected tissue specimens from 46 patients with NPC at the First Affiliated Hospital of Xiamen University. Patients were enrolled between April 2013 and December 2015, and all cases were histologically confirmed as non-keratinizing undifferentiated NPC (WHO III). Staging was determined by physician, according to the seventh edition of the American Joint Committee on Cancer (AJCC) staging of NPC. All tissue specimens were collected before any treatment. Complete blood cell counts (within 30 days before primary treatment) were determined for all patients, and blood parameters from patients with acute conditions, such as bacterial or viral infections, were invalidated. The
dNLR was calculated as neutrophils $\div$ (leukocytes - neutrophils). Demographic, clinical, pathological, and imagology data were also collected.

After diagnosis, we treated stage I and II disease with radiotherapy with or without concurrent chemotherapy cathode ray tube, and we treated stage III and IV disease with cisplatin-based radiochemotherapy regimens and neoadjuvant chemotherapy regimens. The patients received radiotherapy via the intensity-modulated radiation therapy (IMRT) technique, and concurrent cisplatin $\left(100 \mathrm{mg} / \mathrm{m}^{2}\right)$ chemotherapy was administered on days 1, 22, and 43 . Follow-up was performed every 3 months in the first and second years after treatment and every 6 months from the third year and included nasopharynx and neck imaging by MRI and nasopharyngoscopy, chest CT, blood biochemical tests, and a bone scan.

This study was approved by the Ethics Committee of the First Affiliated Hospital of Xiamen University. All participants' consent was both written and informed in the study.

\section{Immunohistochemistry}

Paraffin-embedded NPC tissues were sliced into 4- $\mu$ m-thick sections and stained with hematoxylin and eosin for histology. For immunohistochemical detection of Ki67, the tissue sections were deparaffinized in xylene and rehydrated in a graded series of ethanol followed by microwave treatment in citrate buffer ( $\mathrm{pH} 6.0$ ) for antigen retrieval. Specimens were incubated with a monoclonal anti-Ki67 antibody (MIB-1; $\mathrm{MXB}$ ) at a dilution of $1: 100$ at $4{ }^{\circ} \mathrm{C}$ overnight, according to the manufacturer's instructions. The immunostained tissue sections were scored by two independent investigators who were blinded to the clinical data. The mean percentage of positive cells in at least five high-powered fields was recorded.

\section{Statistical analysis}

All statistical analyses were conducted by using SPSS 22.0 statistical analysis software. Receiver operating characteristic (ROC) curves were generated. For each ROC curve, the cut-off value, which corresponds to a unique sensitivity and specificity, and the critical value, which corresponds to the maximum point of the Jordan index, were calculated. Kaplan-Meier curves were generated to calculate survival rates, followed by the log-rank test. The Cox model was used for multivariate analysis. OS was measured from the first day of treatment until death, and PFS was defined as the period from the first day of treatment until the date of first recurrence or death. Ki67 expression (low vs. high), dNLR (low vs. high), AJCC stage (I or II vs. III or IV), 
primary tumor ( $\mathrm{T}$ ) stage ( $\mathrm{T} 1$ or $\mathrm{T} 2 \mathrm{vs.} \mathrm{T} 3$ or $\mathrm{T} 4$ ), and regional lymph nodes $(\mathrm{N})$ stage (N0-N2 vs. N3) were included in the regression models. The $\chi^{2}, \chi^{2}$ continuity correction, and Fisher's exact test were used to determine the proportion of independents. $P$-values $<0.05$ were considered statistically significant.

\section{Results}

\section{Patients characteristics}

One patient was lost to follow-up and was excluded. The median age of the 45 patients was 47 years old (range, 23-79 years). There were 31 males and 14 females. By staging, $6.67 \%$ of patients had stage I and II disease, while $93.33 \%$ had stage III and IV disease. Of the 45 patients, 14 (31.1\%) were current or former smokers. The median Ki67 expression was $70 \%$ (range, 15-90\%), and the median dNLR was 1.67 (range, 0.56-5.49, Table 1). All patients had histologically confirmed non-keratinizing undifferentiated squamous cell cancer (WHO III). After a median follow-up of 41 months (range, 15-56 months), 11 patients showed disease progression, and 8 patients had died.

\section{Ki67 expression and NPC prognosis}

We plotted a ROC curve to calculate the cutoff value for Ki67 protein expression (77.5\%; the area under the ROC curve was 0.814), which was used to test the association between Ki67

Table I Patient characteristics

\begin{tabular}{lll}
\hline Variable & Number & Percentage \\
\hline Age (years) & & \\
$\quad$ Median (range) & $47(27-79)$ & \\
$\quad<45$ & 19 & 42.2 \\
$\geq 45$ & 26 & 57.8 \\
Gender & & \\
$\quad$ Male & 31 & 68.9 \\
$\quad$ Female & 14 & 31.1 \\
T stage & & \\
TI/T2 & 9 & 20.0 \\
T3/T4 & 36 & 80.0 \\
N stage & & \\
N0-N2 & 34 & 75.6 \\
N3 & 11 & 24.4 \\
Clinical stage & & \\
I/II & 3 & 6.7 \\
III/IV & 42 & 93.3 \\
Smoking history & & 68.9 \\
Nonsmoker & 31 & 31.1 \\
Smoker & 14 & \\
Ki67 expression & & \\
Median (range) & $70 \%($ I5-90\%) \\
dNLR & & \\
Median (range) & 1.67 (0.56-5.49) \\
\hline Abbreviations: dNLR, derived neutrophil-lymphocyte ratio: N, node: T, tumor
\end{tabular}

Cancer Management and Research 2018:10 expression level and OS (Figure 1). We identified tumors with $<77.5 \%$ as low-expression tumors and tumors with $\geq 77.5 \%$ as the high-expression tumors. Among the study patients, there were 29 with low Ki67-expressing tumors and 16 with high Ki67-expressing tumors. We then tested the association between Ki67 protein expression and patient survival using the RECIST 1.1 standard. The OS and PFS of patients with low Ki67-expressing tumors were significantly longer than those of patients with high Ki67-expressing tumors. In the low and high expression groups, the 3-year OS rates were $93.1 \%$ and $62.5 \%$, respectively $(P=0.009)$, and the 3 -year PFS rates were $93.1 \%$ and $56.3 \%$, respectively $(P=0.003$; Figure 2). This result indicates that high Ki67 expression predicts poor prognosis in OS and PFS.

\section{The $d N L R$ and NPC prognosis}

The ROC curve was also used to identify the critical value of the dNLR (2.01; the area under the ROC curve was 0.682). This indicates that a low dNLR is $<2.01$, while a high dNLR is $\geq 2.01$. The numbers of patients in the low and high dNLR groups were 31 and 14 , respectively. We concluded that a low dNLR level was significantly related to improved patient survival. However, while the 3-year OS rate of patients with low dNLR tumors was $90.3 \%$ versus $64.3 \%$ in patients with high dNLR tumors $(P=0.023$; Figure 3$)$, the dNLR did not show any statistical significance for the 3 -year PFS $(P=0.077)$.

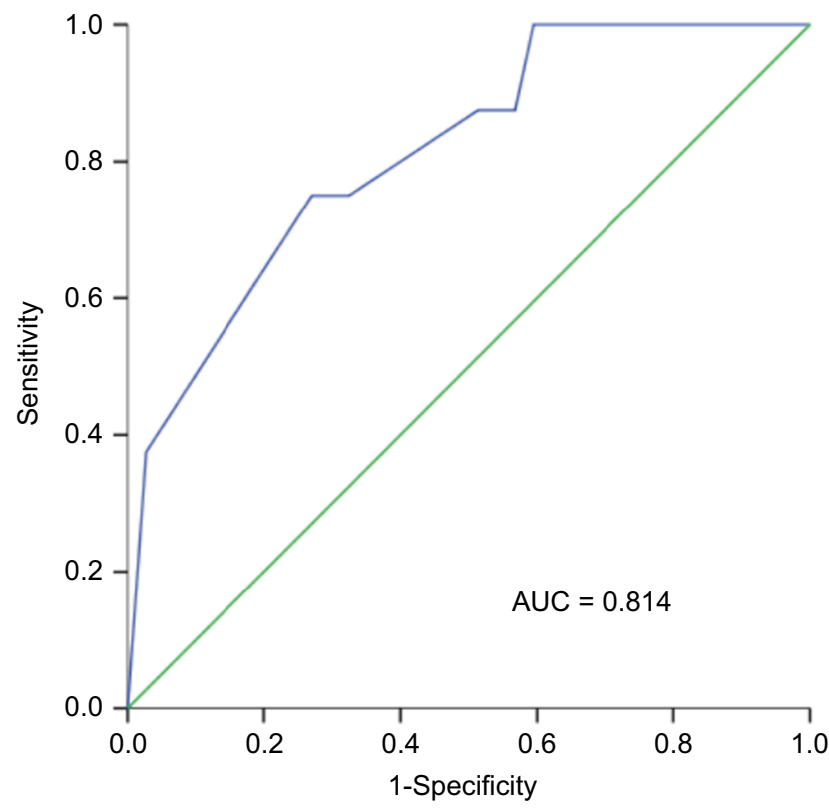

Figure I ROC curve analysis was performed to calculate the optimal cutoff value of Ki67 expression.

Note: The Ki67 expression of the farthest point away from the chance line was 77.5\%.

Abbreviations: AUC, area under curve; ROC, receiver operating characteristic. 


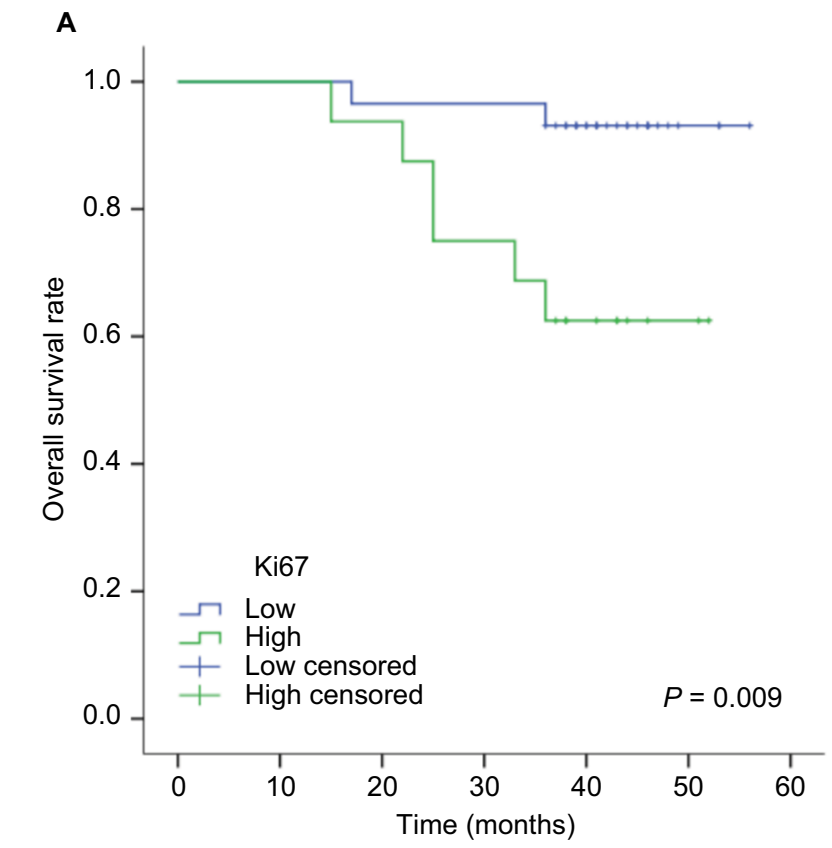

B

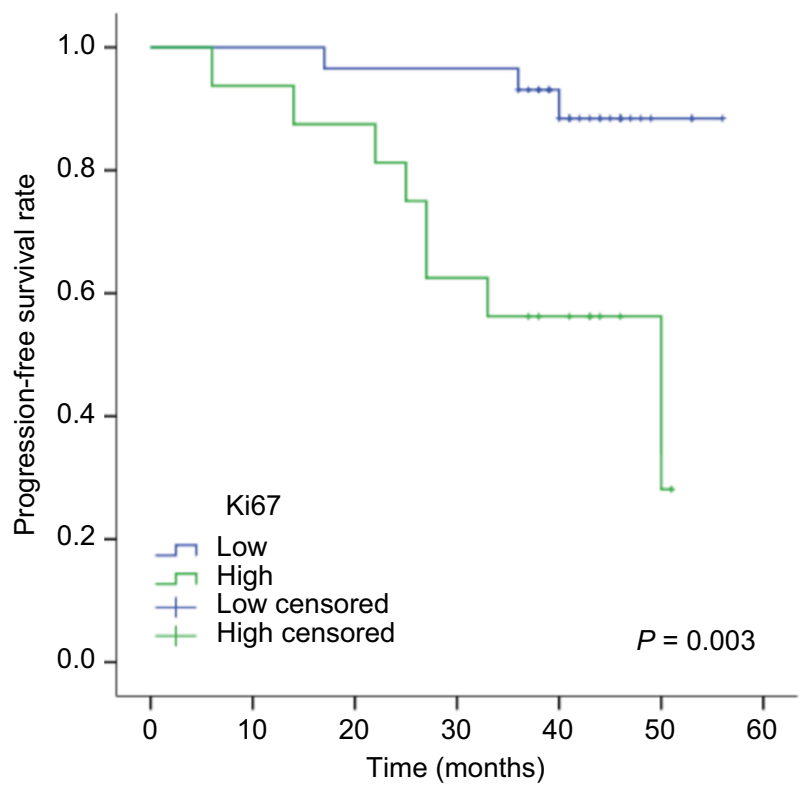

Figure 2 Association of Ki67 expression with overall survival (A) and progression-free survival (B).

A

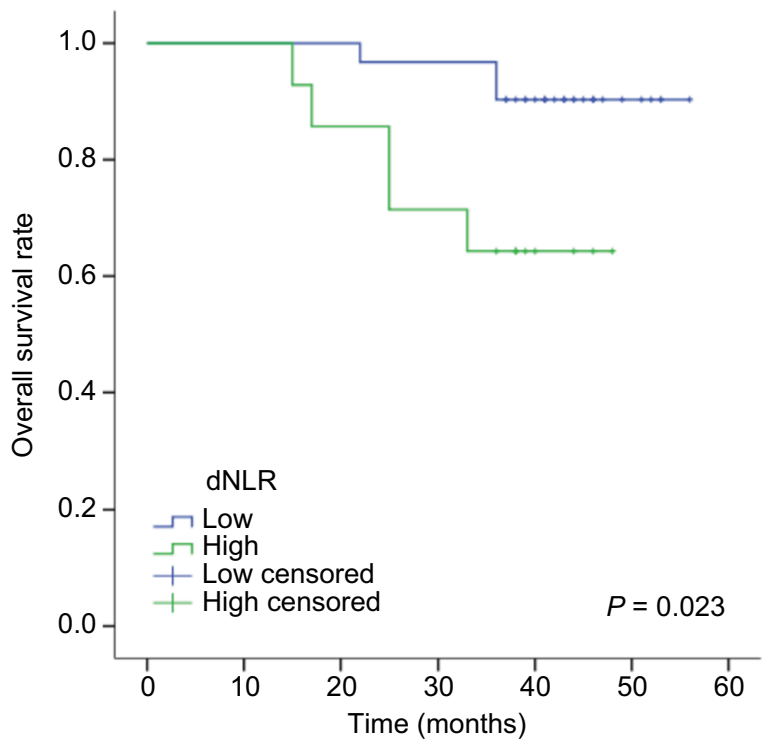

B

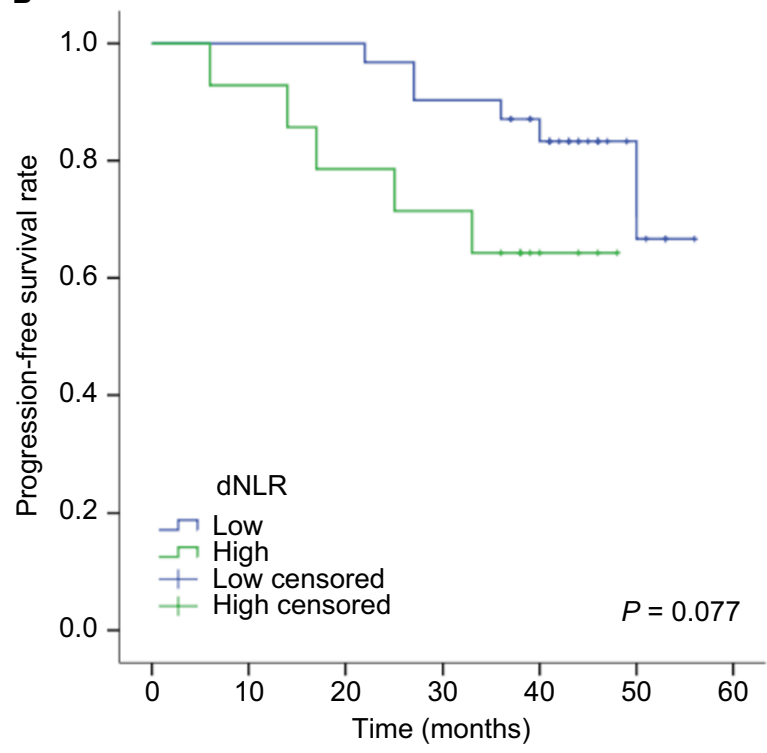

Figure 3 Association of the dNLR with overall survival (A) and progression-free survival (B).

Abbreviation: $\mathrm{dNLR}$, derived neutrophil-lymphocyte ratio.

\section{Correlation of Ki67 expression and the dNLR with disease variables in NPC}

Among the 45 patients, $64.4 \%$ showed low Ki67 expression, while $35.6 \%$ showed high Ki67 expression, and $68.9 \%$ had a low dNLR, while $31.1 \%$ had a high dNLR. There was no statistically significant correlation between Ki67 expression and disease variables. Furthermore, the dNLR was not significantly related to gender, $\mathrm{N}$ stage, clinical stage, or smoking history but was significantly associated with age $(P=0.044)$ and T stage $(P=0.04$; Table 2$)$.

\section{Multivariate analysis of NPC}

Multivariate analysis was performed by the Cox multiple factors regression model, and Ki67 expression, the dNLR, and N stage were independent prognostic factors for OS (Table 3). Since the dNLR was not significantly related to PFS in the 
Table 2 Associations of Ki67 and dNLR with disease variables

\begin{tabular}{|c|c|c|c|c|c|c|}
\hline \multirow[t]{2}{*}{ Variable } & \multicolumn{3}{|l|}{ Ki67 } & \multicolumn{3}{|l|}{ dNLR } \\
\hline & Low & High & $P$-value & Low & High & $P$-value \\
\hline Total & 29 (64.4\%) & $16(35.6 \%)$ & & 31 (68.9\%) & 14 (31.1\%) & \\
\hline Age (years) & & & 0.878 & & & 0.044 \\
\hline$<45$ & 12 (63.2\%) & 7 (36.8\%) & & $10(52.6 \%)$ & 9 (47.4\%) & \\
\hline$\geq 45$ & 17 (65.4\%) & $9(34.6 \%)$ & & 21 (80.8\%) & 5 (19.2\%) & \\
\hline Gender & & & 0.32 & & & 0.253 \\
\hline Male & II (78.6\%) & $3(21.4 \%)$ & & $8(57.1 \%)$ & $6(42.9 \%)$ & \\
\hline Female & 18 (58.1\%) & 13 (4I.9\%) & & $23(74.2 \%)$ & $8(25.8 \%)$ & \\
\hline T stage & & & 1 & & & 0.04 \\
\hline TI/T2 & $6(66.7 \%)$ & $3(33.3 \%)$ & & $9(100 \%)$ & $0(0 \%)$ & \\
\hline T3/T4 & $23(63.9 \%)$ & $13(36.1 \%)$ & & $22(61.1 \%)$ & $22(61.1 \%)$ & \\
\hline $\mathrm{N}$ stage & & & 0.43 & & & 0.954 \\
\hline No-N2 & $23(67.6 \%)$ & II (32.4\%) & & 24 (70.6\%) & 10 (29.4\%) & \\
\hline N3 & $6(54.5 \%)$ & 5 (45.5\%) & & 7 (63.6\%) & $4(36.4 \%)$ & \\
\hline Clinical stage & & & 0.542 & & & $0.54 I$ \\
\hline $\mathrm{I} / \mathrm{II}$ & $3(100 \%)$ & $0(0 \%)$ & & $3(100 \%)$ & $0(0 \%)$ & \\
\hline $\mathrm{III} / \mathrm{IV}$ & 26 (61.9\%) & $16(38.1 \%)$ & & $28(66.7 \%)$ & $14(33.3 \%)$ & \\
\hline Smoking history & & & 0.174 & & & 0.197 \\
\hline Nonsmoker & 22 (7I\%) & $9(29 \%)$ & & 19 (61.3\%) & 12 (38.7\%) & \\
\hline Smoker & $7(50 \%)$ & $7(50 \%)$ & & $12(85.7 \%)$ & $2(14.3 \%)$ & \\
\hline
\end{tabular}

Notes: Data presented as $n$ (\%). Bold figures indicate as statistics significance $(P<0.05)$.

Abbreviations: $\mathrm{dNLR}$, derived neutrophil-lymphocyte ratio; $\mathrm{N}$, node; $\mathrm{T}$, tumor.

Table 3 Multivariate analysis of the Cox risk ratio model for OS

\begin{tabular}{llll}
\hline Parameter & HR & $\mathbf{9 5 \% ~ C l}$ & P-value \\
\hline T stage & 1.334 & $0.111-16.059$ & 0.821 \\
N stage & 0.112 & $0.018-0.696$ & $\mathbf{0 . 0 1 9}$ \\
Smoking history & 0.428 & $0.074-2.474$ & 0.343 \\
Ki67 & 0.116 & $0.014-0.975$ & $\mathbf{0 . 0 4 7}$ \\
dNLR & 0.076 & $0.010-0.559$ & $\mathbf{0 . 0 1 1}$ \\
\hline
\end{tabular}

Note: Bold figures indicate as statistics significance $(P<0.05)$.

Abbreviations: $\mathrm{Cl}$, confidence interval; $\mathrm{dNLR}$, derived neutrophil-lymphocyte ratio; HR, hazard ratio; $\mathrm{N}$, node; OS, overall survival; $\mathrm{T}$, tumor

Kaplan-Meier analysis, we excluded this parameter in the multivariate analysis of PFS. Ki67 expression and N stage were independent prognostic factors for PFS (Table 4). These results indicated that $\mathrm{Ki} 67$ expression, the dNLR, and N stage were independent prognostic factors for OS in patients with NPC, while Ki67 expression and N stage were independent prognostic factors for PFS.

\section{Combined prediction with $\mathrm{Ki} 67$ expression and dNLR of survival in NPC}

Both Ki67 expression and dNLR were independently associated with OS in the Cox proportional hazard model; therefore, we analyzed the combined effect of Ki67 expression and the dNLR on the OS of patients with NPC. Patients were divided into three groups based on $\mathrm{Ki} 67$ expression and dNLR: high risk, both factors were high; intermediate risk, one factor was high; and low risk, neither factor was high. Of the 45 NPC patients, 5 (11.1\%) were at high risk, 20 (44.4\%)
Table 4 Multivariate analysis of the Cox risk ratio model for PFS

\begin{tabular}{llll}
\hline Parameter & HR & $\mathbf{9 5 \%} \mathbf{C l}$ & P-value \\
\hline T stage & 0.969 & $0.198-4.732$ & 0.969 \\
N stage & 0.237 & $0.067-0.842$ & $\mathbf{0 . 0 2 6}$ \\
Smoking history & 0.511 & $0.136-1.926$ & 0.321 \\
Ki67 & 0.203 & $0.051-0.811$ & $\mathbf{0 . 0 2 4}$ \\
\hline
\end{tabular}

Note: Bold figures indicate as statistics significance $(P<0.05)$.

Abbreviations: $\mathrm{Cl}$, confidence interval; $\mathrm{HR}$, hazard ratio; $\mathrm{N}$, node; PFS, progression-free survival; $\mathrm{T}$, tumor.

were at intermediate risk, and 20 (44.4\%) were at low risk. The 3 -year OS rates were $20 \%, 85 \%$, and $95 \%$ for the high, intermediate, and low risk groups, respectively $(P<0.001$; Figure 4). This result indicates that high Ki67 expression and high dNLR predict poor OS.

\section{Discussion}

The clinical outcome of NPC patients has greatly improved, in part due to the application of IMRT and concurrent chemotherapy. ${ }^{17}$ However, the same therapeutic regiment can result in different responses and outcomes in patients with similar clinical characteristics. Therefore, biomarkers for prognosis and the prediction of therapeutic response in NPC patients are greatly needed. Measurement of Ki67 expression and the dNLR is reliable, inexpensive, and easy.

Although there have been numerous studies on Ki67 expression in breast cancer, lymphoma, and other malignant tumors, there have been few studies on the association 


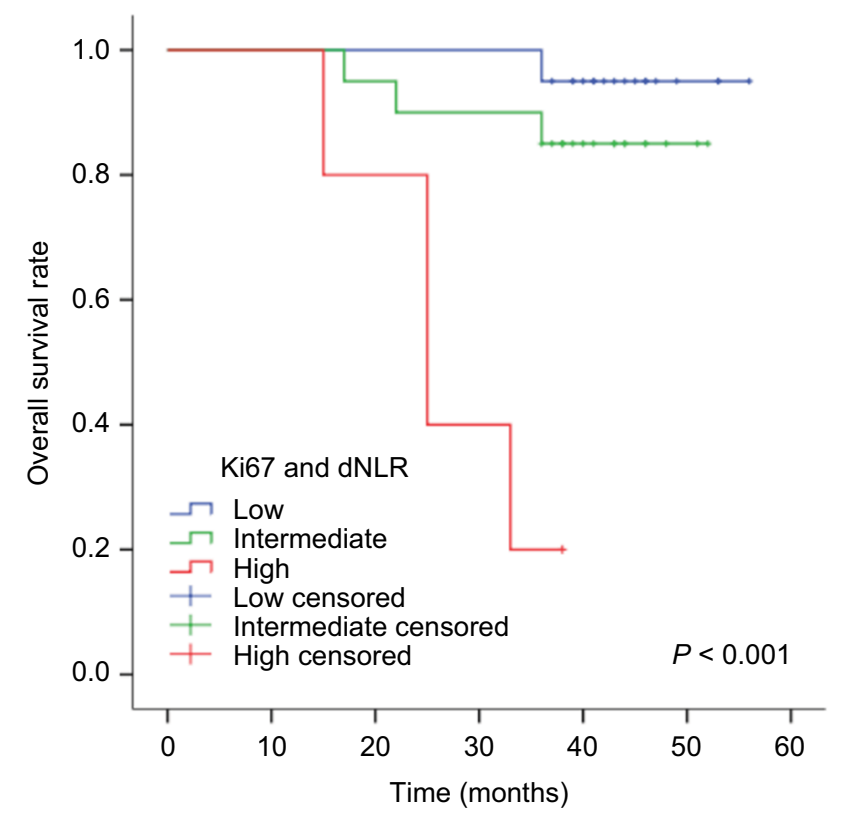

Figure 4 Kaplan-Meier survival curves for NPC patients concerning both Ki67 expression and $\mathrm{dNLR}$.

Abbreviations: $\mathrm{dNLR}$, derived neutrophil-lymphocyte ratio; NPC, nasopharyngeal carcinoma.

between Ki67 expression and the survival in NPC. Our study showed that high Ki67 expression may serve as an indicator of poor prognosis in NPC, consistent with prior reports. ${ }^{18,19}$ Ki67 is a nuclear protein that is tightly linked to the cell cycle, and its expression is intimately related to cell proliferation. ${ }^{4,20}$ Logically, higher Ki67 expression in tumors represents more proliferative activity, therefore, it should be associated with an unfavorable prognosis. High Ki67 expression has also been shown to be associated with adverse clinical outcomes in many malignant tumors., ${ }^{91,22}$ Interestingly, Ki67 is an independent marker in colorectal cancers, and high Ki67 expression is associated with a favorable clinical outcome. ${ }^{10}$ These conflicting results may be due to differences in the thresholds used and the biological characteristics of these diverse tumors. A previous study has demonstrated that the proliferative activity within a tumor is often heterogeneous. ${ }^{23}$ Therefore, researchers have recommended that Ki67 expression be assessed in the tumor invasive margin, which is a zone of high proliferation. ${ }^{7}$ It is questionable whether the Ki67 expression measured in a core needle biopsy obtained in routine clinical practice adequately represents the entire tumor. In our study, expression in $75 \%$ of cells was used as the cut-off for high Ki67 expression. We argue that the Ki67 cut-off may change according to variabilities in patients or tumors, antibodies used for IHC staining, and the investigators. Therefore, there is a substantial unmet need for uniform standard of assay methods and cutoff values. In addition, the detailed relationship between Ki67-defined proliferation and the upstream and downstream biological mechanisms is not clear. A study by Zhao et al showed that high Ki67 expression was correlated with radiation resistance in NPC, ${ }^{18}$ and a study by He et al demonstrated that reduced expression of $B M A L 1$, a biological clock gene, is associated with a high percentage of Ki67 expression and poor OS in NPC. ${ }^{19}$ These studies may explain in part why high Ki67 expression is associated with an unfavorable prognosis in NPC.

Since RudolfVirchow first suggested that "lymphoreticular infiltrate" reflected the origin of cancer at sites of chronic inflammation, the relationship between inflammation and cancer has been explored. Several blood markers have been investigated as indicators of systemic inflammation to predict prognosis in cancer, including dNLR, NLR, albumin, and LDH. However, the levels of albumin, CRP, and LDH are not always examined in clinical practice. However, peripheral blood testing is carried out in day-to-day clinical practice; therefore, the dNLR is easily obtained. A previous study of more than 12,000 patients showed that the dNLR could be used as a potential prognostic indicator for different cancers, ${ }^{16}$ and our study indicated that OS was obviously decreased in patients with a high dNLR. It is of interest that the most commonly used dNLR threshold is $2 .^{16,24}$ In the present study, we used ROC curve analysis and obtained a similar cutoff value. Neutrophils can promote tumor development and inhibit lymphocyte activity. Conversely, lymphocytes are correlated with a better prognosis in cancer patients. ${ }^{25}$ Elevated blood neutrophils have been reported to secrete proangiogenic factors, including matrix metalloproteinase (MMP)-8, MMP-9, IL-8, and vascular endothelial growth factor (VEGF) ${ }^{26,27}$ VEGF contributes to tumor angiogenesis and progression. Moreover, neutrophils can induce immune suppression by inhibiting activated T cells and NK cells. In a study of 1,410 NPC patients, elevated blood neutrophil counts were related to poor PFS and OS. ${ }^{28}$ Lymphocytes are involved in cytokine production and cytotoxic cell death, which inhibit the proliferation and metastasis of tumor cells in anti-tumorigenic responses. ${ }^{29}$ Our aim was to find convenient, inexpensive, and independent prognostic factors for NPC. Other factors, such as albumin, LDH, and CRP, are not routinely measured in day-to-day clinical practice, whereas the dNLR can be easily calculated from a peripheral blood count. An elevated dNLR reflects both a higher neutrophil count and a lower lymphocyte count; therefore, we selected the dNLR as an optimal biomarker to study. The 
consequence of changes in the numbers of neutrophils and lymphocytes is in agreement with the "seed and soil" theory of the nature of cancer progression and metastasis. ${ }^{30}$ There are few reports on the dNLR and prognosis in NPC, and our data in NPC patients confirmed the findings for other tumors, demonstrating that the dNLR has an independent prognostic impact on survival.

According to our research, Ki67 expression levels and the dNLR are promising independent prognostic factors in NPC, and the combination of these two parameters predicted poor clinical outcomes. NPC patients with high Ki67 expression and/or a high dNLR may require more aggressive therapies, such as adjuvant therapy and targeted therapy. Further studies with larger samples groups are needed.

The current study shows encouraging data that Ki67 and dNLR are promising indicators of prognosis in NPC. However, this study has some limitations that need to be addressed. First, it was a single-center study with a limited number of patients, and the follow-up time was insufficient. However, our study was the first to combine Ki67 expression with dNLR to predict survival in NPC, and the trend is obvious in the high Ki67 and high dNLR groups. Future validation of our findings with a larger population and longer follow-up period is warranted. Second, the dNLR might be influenced by many diverse factors, including systemic infection, chronic renal disease, hypertension, and diabetes. ${ }^{31-33}$ Based on our study design, acute conditions, such as bacterial infections, that would influence the dNLR can be excluded. In future studies, procalcitonin and CRP could be analyzed to exclude infectious factors. Nevertheless, in multivariate analysis with the Cox risk ratio model, our data suggest that pretreatment dNLR is an independent prognostic factor in patients with NPC.

\section{Conclusion}

Ki67 expression and the dNLR are the reliable, convenient, inexpensive, and independent prognostic factors for patients with NPC. Combined analysis may be more effective for predicting prognosis, as concurrently high Ki67 and dNLR predict a significantly poor outcome.

\section{Acknowledgment}

This study was supported by a grant from the Natural Science Foundation of Fujian (Grant No. 2016J01633).

\section{Disclosure}

The authors report no conflicts of interest in this work.

\section{References}

1. Lee AW, Ma BB, Ng WT, Chan AT. Management of nasopharyngeal carcinoma: current practice and future perspective. J Clin Oncol. 2015;33(29):3356-3364.

2. Zhang L, Chen QY, Liu H, Tang LQ, Mai HQ. Emerging treatment options for nasopharyngeal carcinoma. Drug Des Devel Ther. 2013;7:37-52

3. Cuylen S, Blaukopf C, Politi AZ, et al. Ki-67 acts as a biological surfactant to disperse mitotic chromosomes. Nature. 2016;535(7611):308-312.

4. Lopez F, Belloc F, Lacombe F, et al. Modalities of synthesis of Ki67 antigen during the stimulation of lymphocytes. Cytometry. 1991;12(1):42-49.

5. Ahn HK, Jung M, Ha SY, et al. Clinical significance of Ki-67 and p53 expression in curatively resected non-small cell lung cancer. Tumour Biol. 2014;35(6):5735-5740.

6. Luporsi E, Andre F, Spyratos F, et al. Ki-67: level of evidence and methodological considerations for its role in the clinical management of breast cancer: analytical and critical review. Breast Cancer Res Treat. 2012;132(3):895-915.

7. Dowsett M, Nielsen TO, A'Hern R, et al. Assessment of Ki67 in breast cancer: recommendations from the International Ki67 in Breast Cancer working group. J Natl Cancer Inst. 2011;103(22):1656-1664.

8. Penault-Llorca F, Andre F, Sagan C, et al. Ki67 expression and docetaxel efficacy in patients with estrogen receptor-positive breast cancer. J Clin Oncol. 2009;27(17):2809-2815.

9. Rasmy A, Abozeed W, Elsamany S, et al. Correlation of preoperative Ki67 and serum CA15.3 levels with outcome in early breast cancers a multi institutional study. Asian Pac J Cancer Prev. 2016;17(7): $3595-3600$.

10. Melling N, Kowitz CM, Simon R, et al. High Ki67 expression is an independent good prognostic marker in colorectal cancer. J Clin Pathol. 2016;69(3):209-214.

11. van Soest RJ, Templeton AJ, Vera-Badillo FE, et al. Neutrophil-tolymphocyte ratio as a prognostic biomarker for men with metastatic castration-resistant prostate cancer receiving first-line chemotherapy: data from two randomized phase III trials. Ann Oncol. 2015;26(4):743-749.

12. Cho H, Hur HW, Kim SW, et al. Pre-treatment neutrophil to lymphocyte ratio is elevated in epithelial ovarian cancer and predicts survival after treatment. Cancer Immunol Immunother. 2009;58(1):15-23.

13. Liao LJ, Hsu WL, Wang CT, et al. Prognostic impact of pre-treatment neutrophil-to-lymphocyte ratio (NLR) in nasopharyngeal carcinoma: a retrospective study of 180 Taiwanese patients. Clin Otolaryngol. 2018;43(2):463-469.

14. Absenger G, Szkandera J, Pichler M, et al. A derived neutrophil to lymphocyte ratio predicts clinical outcome in stage II and III colon cancer patients. Br J Cancer. 2013;109(2):395-400.

15. Lee YY, Choi CH, Kim HJ, et al. Pretreatment neutrophil:lymphocyte ratio as a prognostic factor in cervical carcinoma. Anticancer Res 2012;32(4):1555-1561.

16. Proctor MJ, McMillan DC, Morrison DS, Fletcher CD, Horgan PG, Clarke SJ. A derived neutrophil to lymphocyte ratio predicts survival in patients with cancer. Br J Cancer. 2012;107(4):695-699.

17. Kang M, Long J, Li G, et al. A new staging system for nasopharyngeal carcinoma based on intensity-modulated radiation therapy: results of a prospective multicentric clinical study. Oncotarget. 2016;7(12):15252-15261

18. Zhao Y, Shen L, Huang X, et al. High expression of Ki-67 acts a poor prognosis indicator in locally advanced nasopharyngeal carcinoma Biochem Biophys Res Commun. 2017;494(1-2):390-396.

19. He QY, Jin F, Li YY, et al. Prognostic significance of downregulated BMAL1 and upregulated Ki-67 proteins in nasopharyngeal carcinoma. Chronobiol Int. 2018;35(3):348-357.

20. Tan PH, Bay BH, Yip G, et al. Immunohistochemical detection of Ki67 in breast cancer correlates with transcriptional regulation of genes related to apoptosis and cell death. Mod Pathol. 2005;18(3):374-381. 
21. Goltz D, Montani M, Braun M, et al. Prognostic relevance of proliferation markers (Ki-67, $\mathrm{PHH} 3$ ) within the cross-relation of ERG translocation and androgen receptor expression in prostate cancer. Pathology. 2015;47(7):629-636.

22. Suh Y, Amelio I, Guerrero Urbano T, Tavassoli M. Clinical update on cancer: molecular oncology of head and neck cancer. Cell Death Dis. 2014;5(1):e1018.

23. Yang Z, Tang LH, Klimstra DS. Effect of tumor heterogeneity on the assessment of Ki67 labeling index in well-differentiated neuroendocrine tumors metastatic to the liver: implications for prognostic stratification. Am J Surg Pathol. 2011;35(6):853-860.

24. Gomez D, Morris-Stiff G, Toogood GJ, Lodge JP, Prasad KR. Impact of systemic inflammation on outcome following resection for intrahepatic cholangiocarcinoma. J Surg Oncol. 2008;97(6):513-518.

25. Gooden MJ, de Bock GH, Leffers N, Daemen T, Nijman HW. The prognostic influence of tumour-infiltrating lymphocytes in cancer: a systematic review with meta-analysis. Br J Cancer. 2011;105(1):93-103.

26. Kusumanto YH, Dam WA, Hospers GA, Meijer C, Mulder NH. Platelets and granulocytes, in particular the neutrophils, form important compartments for circulating vascular endothelial growth factor. Angiogenesis. 2003;6(4):283-287.
27. Weitzman SA, Gordon LI. Inflammation and cancer: role of phagocyte-generated oxidants in carcinogenesis. Blood. 1990;76(4): 655-663.

28. He JR, Shen GP, Ren ZF, et al. Pretreatment levels of peripheral neutrophils and lymphocytes as independent prognostic factors in patients with nasopharyngeal carcinoma. Head Neck. 2012;34(12): 1769-1776.

29. Ownby HE, Roi LD, Isenberg RR, Brennan MJ. Peripheral lymphocyte and eosinophil counts as indicators of prognosis in primary breast cancer. Cancer. 1983;52(1):126-130.

30. Fidler IJ, Poste G. The "seed and soil" hypothesis revisited. Lancet Oncol. 2008;9(8):808

31. Okyay GU, Inal S, Onec $\mathrm{K}$, et al. Neutrophil to lymphocyte ratio in evaluation of inflammation in patients with chronic kidney disease. Ren Fail. 2013;35(1):29-36.

32. Demir M. The relationship between neutrophil lymphocyte ratio and non-dipper hypertension. Clin Exp Hypertens. 2013;35(8):570-573.

33. Lee GK, Lee LC, Chong E, et al. The long-term predictive value of the neutrophil-to-lymphocyte ratio in type 2 diabetic patients presenting with acute myocardial infarction. QJM. 2012;105(11):1075-1082.
Cancer Management and Research

\section{Publish your work in this journal}

Cancer Management and Research is an international, peer-reviewed open access journal focusing on cancer research and the optimal use of preventative and integrated treatment interventions to achieve improved outcomes, enhanced survival and quality of life for the cancer patient. The manuscript management system is completely online and includes
Dovepress

a very quick and fair peer-review system, which is all easy to use. Visit $\mathrm{http}: / / \mathrm{www}$.dovepress.com/testimonials.php to read real quotes from published authors. 